\title{
NALLD Market
}

The NALLD Market provides a service for lab directors and schools faced with the problem of disposing of master tapes for texts no longer used and equipment no longer needed. The NALLD Journal will list such tapes and equipment for sale by schools. Only tapes sold by the publisher to the school and still in the original package will be listed.

Please note that NALLD and the NALLD Journal accept no responsibility for the transportation of items between buyer and seller. The quality of the materials and the method of payment for them should be a matter established between the buyer and seller before the transaction is made.

The listings in the Journal will be determined by the space available. The NALLD Journal reserves the right to select listings.

Information for tapes offered for sale should be presented in the following form in the order indicated:

Language, title, author, editor, publisher, year, number of reels, number of tracks, recording speed, original cost, selling price (we suggest 50 percent discount), person to contact.

Equipment for sale should be listed as follows in the order indicated:

Type of equipment, manufacturer, description of pertinent features as applicable (as briefly and concisely as possible), condition, year of purchase, original cost, selling price, person to contact.

Listings for the NALLD Market should be sent to C. P. Richardson, Editor, NALLD Journal, Ellis Hall 26-A, Ohio University, Athens, Ohio 45701.

\section{TAPES}

\section{GERMAN}

FOCUS ON GERMAN FOR BEGINNERS - Leroy R. Shaw, Harper and Row, 1965; thirty 5 -inch reels, $33 / 4$, full track. Cost, $\$ 185$; selling price, $\$ 75$.

CONTACT: David A. Hanson, Department of Germanic and Slavic Languages, Brandeis University, Waltham, MA 02154 
NALLD Market

DEUTSCH-ERSTES BUCH - Hugo Mueller, Bruce Publishing Co., single-volume edition (purchased 1971), fifteen 7 -inch reels, $71 / 4$ ips, half track. Cost, $\$ 150$; selling price, $\$ 60$.

CONTACT: Same as above.

\section{SPANISH}

PARA EMPEZAR, Lagrone, Holt, 1964; seven reels, dual track, 7 1/2 ips; original cost, $\$ 70$; selling price, $\$ 15$.

CONTACT: Harold L. Beckman, Chairman, Foreign Language Department, Lincoln High School, Manitowoc, WI 54220.

PARA CONTINUAR, Lagrone, Holt, 1965; nine reels, dual track, 3 3/4 ips; original cost, $\$ 80$; selling price, $\$ 20$.

CONTACT: Same as above.

TERCER CURSO, Holt, 1967; six reels, $33 / 4$ ips; original cost, $\$ 60$; selling price, $\$ 25$.

CONTACT: Same as above.

ESPANOL MODERNO II, Brady, Merrill; eight reels, 3 3/4 ips; original cost, $\$ 90$; selling price, $\$ 20$.

CONTACT: Same as above.

PROGRESSIVE AUDIO LINGUAL TAPES, Regents Publishing Co.; (Drills on grammatical structures, one structure per 5-inch tape), $33 / 4$ ips; Includes tapes 2.41 inclusive, plus tapes $43,50,51,56$, $57,61,70,71,72,73,75,77,78$, and 81 (54 tapes in all); original cost, approximately $\$ 75$; selling price $\$ 20$.

CONTACT: Same as above.

\section{FRENCH}

PROGRESSIVE AUDIO LINGUAL TAPES, Regents Publishing Co.; (Drills on grammatical structures, one structure per 5-inch tape), $33 / 4$ ips; tapes 1-30 inclusive, and 34-90 (87 tapes in all); original cost, approximately $\$ 110$; selling price, $\$ 40$.

CONTACT: Harold L. Beckman, Chairman, Foreign Language Department, Lincoln High School, Manitowoc, WI 54220.

VOIX ET IMAGES DE FRANCE, Center for Curriculum Development, Inc., complete curriculum including 32 filmstrips, 32 tapes, 16 student records, teacher's manuals, student's book, exercise book, etc. These materials are in like-new condition. Original cost, approximately $\$ 175$; selling price, $\$ 60$.

CONTACT: Same as above. 